\title{
Preparation of $\beta$-Enamino Carbonylic Compounds using Microwave Radiation/K-10
}

\author{
Hugo T. S. Braibante*, Mara E. F. Braibante*, Giovanni B. Rosso and Daniela A. Oriques \\ Departamento de Química - CCNE, Universidade Federal de Santa Maria, 97105-900 Santa Maria - RS, Brazil
}

\begin{abstract}
A preparação de uma série de $\beta$-enamino cetonas e ésteres empregando a metodologia de reações em suporte sólido associada à irradiação de microondas é descrita. As reações dos compostos $\beta$-dicarbonílicos cíclicos, acíclicos e $\alpha$-cloro-substituídos, frente a aminas primárias ou aos acetatos de amônio correspondentes, foram efetuadas usando K-10 como suporte sólido e forno de microondas doméstico como fonte de irradiação, obtendo-se os $\beta$-enamino compostos com ótimos rendimentos.
\end{abstract}

The preparation of $\beta$-enamino ketones and esters using the methodology of the reactions on solid support coupled with microwave irradiation is described. The reaction of cyclic, acyclic and $\alpha$-chloro-substituted $\beta$-dicarbonyl compounds with amines or their corresponding ammonium acetates was carried out using K-10 as solid support and a domestic microwave oven as the radiation source to give $\beta$-enamino carbonylic compounds in good yields.

Keywords: enaminones, montmorillonite K-10, microwave irradiation

\section{Introduction}

As part of our program related to the study of the synthesis and reactivity of $\beta$-enamino carbonylic compounds in heterogeneous media, we report now a simple and very fast procedure for the synthesis of these compounds using K-10 in domestic microwave oven (MW).

One of most accepted advances in chemistry methods in recent years is the use of microwave-assisted chemistry. ${ }^{1}$ The effects usually observed with microwave activation in organic reaction are: decreasing reaction times, increase yield, cleaner reaction with easier work-up. "Solvent-free" conditions allow the use of open vessels in domestic microwave ovens. Domestic microwave ovens have the risk of high-pressure incidents, including explosion. One effective method for avoiding this problem is to omit the solvent from the reaction and perform the reaction on solid supports such as clays $-\mathrm{K}-10$. Generally reactions without solvent have been performed at room temperature with poor thermal diffusion of the inorganic solids. ${ }^{2}$ Inorganic oxides such as clays, alumina and silica do not absorb microwaves at $2450 \mathrm{MHz}$ and so are not obstacles for the transmission of microwave irradiation. In a solventless reaction, polar groups and organic compounds present on

*e-mail: hugots@quimica.ufsm.br

This paper is dedicated to Prof. Albert J. Kascheres on occasion of his $60^{\text {th }}$ birthday the surface of the clays strongly absorb microwave irradiation and thus are selectively activated.

Our interest in developing simple and efficient methodologies to obtain $\beta$-enamino carbonylic compounds is due to their nature to function both as ambident nucleophiles and as ambident electrophiles. In this way, enaminones serve as very important intermediates in the synthesis of natural products ${ }^{3}$ and heterocyclic compounds. ${ }^{4,5}$ Anticonvulsivant activity of several secondary enaminones has been reported. ${ }^{6}$ Scott et al. ${ }^{7}$ identified several relationships between chemical features and activity: (i) the separation between the amino group and the aromatic ring should not exceed two methylene units; (ii) the presence of a carbomethoxy group enhances activity; and (iii) the cyclic enaminones are generally more active than the acyclic ones, the restrict conformation provides a more rigid frame of interaction.

\section{Results and Discussion}

Many methods have been described for the preparation of $\beta$-enamino carbonylic compounds, such as condensation of amines with $\beta$-dicarbonylic compounds in aromatic solvents with azeotropic water removal ${ }^{8}$ and hydrogenation (Raney-Ni) of isoxazoles. ${ }^{9}$ Each method has specific applications and limitations such as requiring autoclaves or applying elevated temperature..$^{10}$ The use of low boiling amines is particularly problematic. We have reported ${ }^{11} \mathrm{a}$ 
simple and selective method using montmorillonite K-10 as solid support to obtain a series of $\beta$-enamino ketones and $\beta$-enamino esters. This versatile method has been developed using reagents dispersed in K-10 stirred at room temperature for 15-24 h. Recently, we used the same methodology, but with sonication ${ }^{12}$ for the preparation of a series of $p$-phenyl substituted $\beta$-enamino ketones and $\beta$-enamino derivatives of 2 -acylcycloalkanones. The preparation of enamino ketones without solvent coupled with microwave irradiation using acetylacetone and high boiling point amines has been mentioned in the literature. ${ }^{13}$ For low boiling point amines, we have used their ammonium acetate derivatives and successfully applied our previously developed methodology of reactions on solid support under microwave irradiation for the preparation of $\beta$-enamino carbonylic compounds.

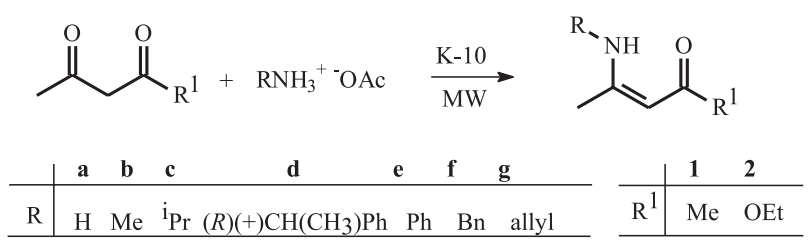

Scheme 1.

4-Amino substituted-3-penten-2-ones 1a-g and ethyl 3-amino substituted-2-butenoates 2a-f, were prepared using K-10 in "solvent-free" conditions under microwave irradiation (Scheme 1). The reaction time required was 3 min except for 1-2a,b and $\mathbf{g}$, which was $5 \mathrm{~min}$. The yields of these compounds were 70-97\%, an improvement when compared to the previously described procedure without microwave radiation. ${ }^{11}$ The stereochemistry of compounds $\mathbf{1}$ and $\mathbf{2}$ is $Z$, as shown by the intramolecular hydrogen bonding in the ${ }^{1} \mathrm{H}$ NMR spectra that contain a typical N-H chemical shifts (8.45-12.47 ppm).

These good results obtained encouraged us to apply this methodology to prepare other $\alpha$-substituted $\beta$-enamino carbonylic compounds and $\beta$-enamino ketones with fixed configurations. Thus for the preparation of the $\alpha$-halo $\beta$-enamino carbonylic compounds we used the reaction of ethyl-2-chloro-acetoacetate (3) and the salts of corresponding amines supported in K-10 under microwave irradiation (see Experimental) to afford ethyl-(3-amino substituted)-2-chloro-2-butenoates 4a-f. We tested this reaction in different times of microwave irradiation and determined the optimal time to be $3 \mathrm{~min}$ (Scheme 2). In the absence of microwave irradiation, the reaction was stirred at room temperature for $24 \mathrm{~h}$ resulting in a large amount of the starting material. For compounds $\mathbf{4 a}, \mathbf{e}$ and $\mathbf{f}$, a mixture of $E / Z$ isomers was observed based in the ${ }^{1} \mathrm{H}$ NMR spectra which showed N-H chemical shifts (9.04-10.04 ppm for $E$ and 5.02-5.90 ppm for $Z$ configuration) different from those observed for $\mathbf{1}$ and $\mathbf{2}$ (see Table 1). ${ }^{11}$

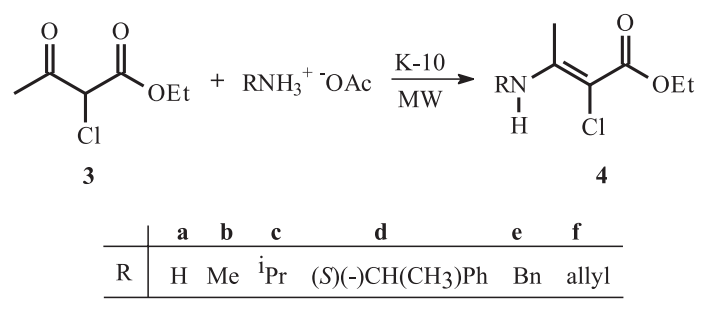

\begin{tabular}{|ccccc|}
\hline Reaction & \multicolumn{4}{c|}{ Yield \% } \\
time (min) & 4a & 4b & 4c & 4e \\
\hline 2 & 75 & 67 & 78 & 92 \\
3 & 83 & 49 & 83 & 97 \\
5 & 79 & 19 & 71 & 92 \\
7 & 83 & 9 & 54 & 79 \\
\hline
\end{tabular}

Scheme 2.

For the preparation of the $\beta$-enamino ketones with fixed configurations, the cyclic 1,3-diketone (dimedone) $\mathbf{5}$ was chosen. The literature describes that dimedone enaminones

Table 1. Ethyl-3-amino substituted-2-chloro-2-butenoates 4a-g prepared

\begin{tabular}{|c|c|c|c|}
\hline Compound & Yield $(\%)$ & ${ }^{1} \mathrm{H} \mathrm{NMR}\left(\mathrm{CDCl}_{3} / \mathrm{TMS}\right) \delta(\mathrm{ppm}), J(\mathrm{~Hz})$ & ${ }^{13} \mathrm{C} \mathrm{NMR}\left(\mathrm{CDCl}_{3} / \mathrm{TMS}\right) \delta(\mathrm{ppm})$ \\
\hline $4 \mathbf{a}$ & 83 & $\begin{array}{l}1.32(\mathrm{t}, J 7.2,3 \mathrm{H}) ; 2.14(\mathrm{~s}, 3 \mathrm{H}) ; 4.21(\mathrm{q}, J 7.2,2 \mathrm{H}) \\
5.02(\mathrm{br}, 1 \mathrm{H}, \mathrm{N}-\mathrm{H}) ; 9.24(\mathrm{br}, 1 \mathrm{H}, \mathrm{N}-\mathrm{H})\end{array}$ & $\begin{array}{l}14.6\left(\mathrm{CH}_{3}\right) ; 22.3\left(\mathrm{CH}_{3}\right) ; 60.5\left(\mathrm{CH}_{2}\right) ; 90.3(\mathrm{C}) ; \\
157.7(\mathrm{C}) ; 164.9(\mathrm{C}=\mathrm{O}) .\end{array}$ \\
\hline $4 b$ & 67 & $\begin{array}{l}1.31(\mathrm{t}, J 7.2,3 \mathrm{H}) ; 2.18(\mathrm{~s}, 3 \mathrm{H}) ; 2.94(\mathrm{~d}, J 5.2,3 \mathrm{H}) \\
4.19(\mathrm{q}, J 7.2,2 \mathrm{H}) ; 9.04(\mathrm{br}, 1 \mathrm{H}, \mathrm{N}-\mathrm{H})\end{array}$ & $\begin{array}{l}14.3\left(\mathrm{CH}_{3}\right) ; 16.1\left(\mathrm{CH}_{3}\right) ; 30.3\left(\mathrm{CH}_{3}\right) ; 59.9\left(\mathrm{CH}_{2}\right) ; \\
88.1(\mathrm{C}) ; 160.7(\mathrm{C}) ; 167.4(\mathrm{C}=\mathrm{O}) .\end{array}$ \\
\hline $4 c$ & 83 & $\begin{array}{l}1.22(\mathrm{~d}, J 6.4,6 \mathrm{H}) ; 1.31(\mathrm{t}, J 7.2,3 \mathrm{H}) ; 2.20(\mathrm{~s}, 3 \mathrm{H}) \\
3.72(\mathrm{~m}, 1 \mathrm{H}) ; 4.19(\mathrm{q}, J 7.2,2 \mathrm{H}) ; 10.04(\mathrm{br}, 1 \mathrm{H}, \mathrm{N}-\mathrm{H})\end{array}$ & $\begin{array}{l}13.8\left(\mathrm{CH}_{3}\right) ; 19.6\left(\mathrm{CH}_{3}\right) ; 26.2\left(\mathrm{CH}_{3}\right) ; 45.4(\mathrm{CH}) \\
60.0\left(\mathrm{CH}_{2}\right) ; 88.2(\mathrm{C}) ; 159.0(\mathrm{C}) ; 167.4(\mathrm{C}=\mathrm{O}) .\end{array}$ \\
\hline 4d & 77 & $\begin{array}{l}1.33(\mathrm{t}, J 7.2,3 \mathrm{H}) ; 1.50(\mathrm{~d}, J 6.8,3 \mathrm{H}) ; 2.00(\mathrm{~s}, 3 \mathrm{H}) \\
4.22(\mathrm{q}, J 7.2,2 \mathrm{H}) ; 4.64(\mathrm{q}, J 6.8,1 \mathrm{H}) ; 7.23-7.35 \\
\left(\mathrm{br}, 5 \mathrm{H}_{\text {arom }}\right) ; 9.52(\mathrm{br}, 1 \mathrm{H}, \mathrm{N}-\mathrm{H})\end{array}$ & $\begin{array}{l}14.5\left(\mathrm{CH}_{3}\right) ; 16.4\left(\mathrm{CH}_{3}\right) ; 23.9\left(\mathrm{CH}_{3}\right) ; 52.7(\mathrm{CH}) ; \\
60.1\left(\mathrm{CH}_{2}\right) ; 89.5(\mathrm{C}) ; 126.5(\mathrm{CH}) ; 127.1(\mathrm{CH}) ; \\
128.3(\mathrm{CH}) ; 143.9(\mathrm{C}) ; 159.4(\mathrm{C}) ; 167.4(\mathrm{C}=\mathrm{O}) .\end{array}$ \\
\hline $4 e$ & 97 & $\begin{array}{l}1.32(\mathrm{t}, J 7.2,3 \mathrm{H}) ; 2.14(\mathrm{~s}, 3 \mathrm{H}) ; 4.21(\mathrm{q}, J 7.2,2 \mathrm{H}) \\
4.44(\mathrm{~d}, J 6.2,2 \mathrm{H}) ; 5.90 \text { (isomer } Z, \text { br, } 1 \mathrm{H}, \mathrm{N}-\mathrm{H}) \\
\left.7.12-7.34\left(\mathrm{br}, 5 \mathrm{H}_{\text {arom }}\right) ; 9.50 \text { (isomer } E, \text { br, } 1 \mathrm{H}, \mathrm{N}-\mathrm{H}\right)\end{array}$ & $\begin{array}{l}14.4\left(\mathrm{CH}_{3}\right) ; 16.5\left(\mathrm{CH}_{3}\right) ; 47.6\left(\mathrm{CH}_{2}\right) ; 60.1\left(\mathrm{CH}_{2}\right) ; \\
89.5(\mathrm{C}) ; 126.6(\mathrm{CH}) ; 127.1(\mathrm{CH}) ; 128.7(\mathrm{CH}) ; \\
138.1(\mathrm{C}) ; 159.8(\mathrm{C}) ; 167.4(\mathrm{C}=\mathrm{O}) .\end{array}$ \\
\hline $4 f$ & 80 & $\begin{array}{l}1.33(\mathrm{t}, J 7.2,3 \mathrm{H}) ; 2.16(\mathrm{~s}, 3 \mathrm{H}) ; 3.87(\mathrm{~m}, 2 \mathrm{H}) ; 4.20 \\
(\mathrm{q}, J 7.2,2 \mathrm{H}) ; 5.20(\mathrm{~m}, 2 \mathrm{H}) ; 5.67(\mathrm{~m}, 1 \mathrm{H}) ; 5.70 \\
\text { (isomer } Z, \mathrm{br}, 1 \mathrm{H}, \mathrm{N}-\mathrm{H}) ; 9.22(\text { isomer } E, \mathrm{br}, 1 \mathrm{H}, \mathrm{N}-\mathrm{H})\end{array}$ & $\begin{array}{l}14.8\left(\mathrm{CH}_{3}\right) ; 16.5\left(\mathrm{CH}_{3}\right) ; 45.5\left(\mathrm{CH}_{2}\right) ; 60.2\left(\mathrm{CH}_{2}\right) ; \\
89.4(\mathrm{C}) ; 116.6\left(\mathrm{CH}_{2}\right) ; 134.6(\mathrm{CH}) ; 160.3(\mathrm{C}) ; \\
167.8(\mathrm{C}=\mathrm{O}) .\end{array}$ \\
\hline
\end{tabular}


have a complex reactivity that depends on the conditions employed,${ }^{14}$ which emphasizes the importance of the preparation of these compounds without by-products. The reaction of 5,5-dimethylcyclohex-2-en-1-one (5) with ammonium acetates supported on K-10 was carried out under microwave irradiation to give 3-amino substituted-5,5dimethylcyclohex-2-enones 6a-g in good yields (Scheme 3 , Table 2). It is reported ${ }^{8}$ that the reaction of the dimedone with amines in refluxing xylenes gives, in addition to the expected enaminone the corresponding dienamine-dione but with our methodology this was not observed.

To summarize, the microwave irradiation in "solvent-free" conditions with montmorillonite K-10 in open vessels provides an easy and efficient procedure to obtain different types of the $\beta$-enamino carbonylic compounds in good yield and with much shorter time periods than the conventional procedure and it can be applied on different scales. The microwave/K-10 methodology is a clean and efficient process from both economic and environmental standpoint and with the essential characteristics of a green technology.

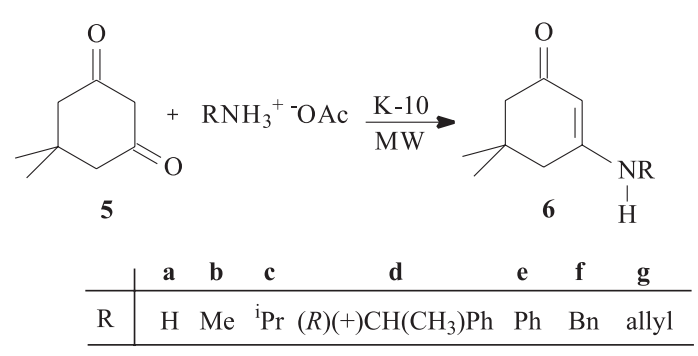

Scheme 3.

\section{Experimental}

Melting points were determined with a Microquímica APF-301 apparatus and are uncorrected. ${ }^{1} \mathrm{H}$ and ${ }^{13} \mathrm{C}$ NMR spectra were recorded on a Bruker DPX-200 spectrometer in $\mathrm{CDCl}_{3}$ /TMS. Elemental analyses were carried out on a Vario CHN-standard analyzer. Microwave irradiations were carried out in a SANYO EM-700T domestic oven (700 W).

Table 2. 3-Amino substituted-5,5-dimethylcyclohex-2-en-1-ones 6a-g prepared

\begin{tabular}{|c|c|c|c|c|c|c|c|c|c|}
\hline Compound & $\begin{array}{l}\text { Reaction } \\
\text { time (min) }\end{array}$ & $\frac{\mathrm{mp}}{\left({ }^{\circ} \mathrm{C}\right)}$ & $\begin{array}{l}\text { Yield } \\
(\%)\end{array}$ & $\begin{array}{l}\text { Molecular } \\
\text { Formula }\end{array}$ & $\begin{array}{l}\text { Ana } \\
\text { Calc } \\
\text { C }\end{array}$ & $\begin{array}{l}\text { alysis( } \\
\text { cd./For } \\
\text { H }\end{array}$ & $\begin{array}{l}(\%) \\
\mathrm{und} \\
\mathrm{N}\end{array}$ & $\begin{array}{l}{ }^{1} \mathrm{H} \mathrm{NMR}\left(\mathrm{CDCl}_{3} / \mathrm{TMS}\right) \\
\delta(\mathrm{ppm}), J(\mathrm{~Hz})\end{array}$ & $\begin{array}{l}{ }^{13} \mathrm{C} \mathrm{NMR}\left(\mathrm{CDCl}_{3} / \mathrm{TMS}\right) \\
\delta(\mathrm{ppm})\end{array}$ \\
\hline $6 a$ & 3 & 168 & 90 & $\begin{array}{l}\mathrm{C}_{8} \mathrm{H}_{13} \mathrm{NO} \\
139.19\end{array}$ & $\begin{array}{l}69.00 \\
68.93\end{array}$ & $\begin{array}{l}9.40 \\
9.03\end{array}$ & $\begin{array}{l}10.10 \\
10.01\end{array}$ & $\begin{array}{l}0.96(\mathrm{~s}, 6 \mathrm{H}) ; 1.91(\mathrm{~s}, 2 \mathrm{H}) \\
2.12(\mathrm{~s}, 2 \mathrm{H}) ; 4.91(\mathrm{~s}, 1 \mathrm{H}) \\
6.68\left(\mathrm{br}, 2 \mathrm{H}, \mathrm{NH}_{2}\right)\end{array}$ & $\begin{array}{l}28.0\left(\mathrm{CH}_{3}\right) ; 32.2\left(\mathrm{CH}_{2}\right) ; 41.6(\mathrm{C}) ; \\
49.8\left(\mathrm{CH}_{2}\right) ; 95.9(\mathrm{CH}) ; 165.3(\mathrm{C}) ; \\
193.8(\mathrm{C}=\mathrm{O}) .\end{array}$ \\
\hline $6 b$ & 2 & 158 & 47 & $\begin{array}{l}\mathrm{C}_{9} \mathrm{H}_{15} \mathrm{NO} \\
153.22\end{array}$ & $\begin{array}{l}70.50 \\
70.18\end{array}$ & $\begin{array}{l}9.90 \\
9.58\end{array}$ & $\begin{array}{l}9.10 \\
9.09\end{array}$ & $\begin{array}{l}1.07(\mathrm{~s}, 6 \mathrm{H}) ; 2.17(\mathrm{~s}, 2 \mathrm{H}) \\
2.22(\mathrm{~s}, 2 \mathrm{H}) ; 2.80(\mathrm{~d}, J 6.0 \\
3 \mathrm{H}) ; 5.07(\mathrm{~s}, 1 \mathrm{H}) ; 5.70 \\
(\mathrm{br}, 1 \mathrm{H}, \mathrm{N}-\mathrm{H})\end{array}$ & $\begin{array}{l}28.2\left(\mathrm{CH}_{3}\right) ; 29.5\left(\mathrm{CH}_{3}\right) ; 32.7\left(\mathrm{CH}_{2}\right) ; \\
43.1(\mathrm{C}) ; 50.2\left(\mathrm{CH}_{2}\right) ; 94.6(\mathrm{CH}) ; \\
162.5(\mathrm{C}) ; 196.1(\mathrm{C}=\mathrm{O}) .\end{array}$ \\
\hline $6 c$ & 2 & 147 & 92 & $\begin{array}{c}\mathrm{C}_{11} \mathrm{H}_{19} \mathrm{NO} \\
181.27\end{array}$ & $\begin{array}{l}72.90 \\
72.58\end{array}$ & $\begin{array}{l}10.60 \\
10.28\end{array}$ & $\begin{array}{l}7.70 \\
7.64\end{array}$ & $\begin{array}{l}1.06(\mathrm{~s}, 6 \mathrm{H}) ; 1.22(\mathrm{~d}, J 6.0 \\
6 \mathrm{H}) ; 2.16(\mathrm{~s} \mathrm{br} 2 \mathrm{H}) ; 2.22 \\
(\mathrm{~s} \mathrm{br}, 2 \mathrm{H}) ; 3.60(\mathrm{~m}, 1 \mathrm{H}) ; 5.09 \\
(\mathrm{~s}, 1 \mathrm{H}) ; 5.83(\mathrm{br}, 1 \mathrm{H}, \mathrm{N}-\mathrm{H})\end{array}$ & $\begin{array}{l}21.8\left(\mathrm{CH}_{3}\right) ; 28.0\left(\mathrm{CH}_{3}\right) ; 32.5\left(\mathrm{CH}_{2}\right) \text {; } \\
43.2(\mathrm{C}) ; 43.9(\mathrm{CH}) ; 50.1\left(\mathrm{CH}_{2}\right) ; \\
94.6(\mathrm{CH}) ; 162.5(\mathrm{C}) ; 196.1(\mathrm{C}=\mathrm{O}) .\end{array}$ \\
\hline 6d & 8 & 146 & 64 & $\begin{array}{l}\mathrm{C}_{16} \mathrm{H}_{21} \mathrm{NO} \\
243.34\end{array}$ & $\begin{array}{l}79.00 \\
78.68\end{array}$ & $\begin{array}{l}8.70 \\
8.89\end{array}$ & $\begin{array}{l}5.80 \\
5.72\end{array}$ & $\begin{array}{l}0.99(\mathrm{~s}, 3 \mathrm{H}) ; 1.05(\mathrm{~s}, 3 \mathrm{H}) ; 1.42 \\
(\mathrm{~d}, J 8.0,3 \mathrm{H}) ; 2.10(\mathrm{~s}, 2 \mathrm{H}) ; \\
2.21(\mathrm{~s}, 2 \mathrm{H}) ; 4.44(\mathrm{q}, J 7.2 \\
J 8.0,1 \mathrm{H}) ; 4.95(\mathrm{~s}, 1 \mathrm{H}) ; 5.92 \\
(\mathrm{~d}, J 7.2,1 \mathrm{H}, \mathrm{N}-\mathrm{H}) ; 7.17-7.34 \\
\left(\mathrm{br}, 5 \mathrm{H}_{\text {arom }}\right)\end{array}$ & $\begin{array}{l}23.3\left(\mathrm{CH}_{3}\right) ; 28.1\left(\mathrm{CH}_{3}\right) ; 32.7\left(\mathrm{CH}_{2}\right) \text {; } \\
43.1(\mathrm{C}) ; 50.2(\mathrm{CH}) ; 52.7\left(\mathrm{CH}_{2}\right) ; \\
96.7(\mathrm{CH}) ; 125.5(\mathrm{CH}) ; 127.2(\mathrm{CH}) \text {; } \\
128.6(\mathrm{CH}) ; 142.9(\mathrm{C}) ; 162.2(\mathrm{C}) \text {; } \\
196.6(\mathrm{C}=\mathrm{O}) .\end{array}$ \\
\hline $6 e$ & 12 & 185 & 90 & $\begin{array}{l}\mathrm{C}_{14} \mathrm{H}_{17} \mathrm{NO} \\
215.24\end{array}$ & $\begin{array}{l}78.10 \\
77.88\end{array}$ & $\begin{array}{l}7.96 \\
7.99\end{array}$ & $\begin{array}{l}6.51 \\
6.51\end{array}$ & $\begin{array}{l}1.05(\mathrm{~s}, 6 \mathrm{H}) ; 2.15(\mathrm{~s}, 2 \mathrm{H}) \\
2.35(\mathrm{~s}, 2 \mathrm{H}) ; 5.53(\mathrm{~s}, 1 \mathrm{H}) \\
7.08-7.30\left(\mathrm{br}, 5 \mathrm{H}_{\text {arom }}\right) ; 8.09 \\
(\mathrm{br}, 1 \mathrm{H}, \mathrm{N}-\mathrm{H})\end{array}$ & $\begin{array}{l}28.0\left(\mathrm{CH}_{3}\right) ; 32.6\left(\mathrm{CH}_{2}\right) ; 42.9(\mathrm{C}) ; \\
50.2\left(\mathrm{CH}_{2}\right) ; 97.2(\mathrm{CH}) ; 123.7(\mathrm{CH}) ; \\
125.1(\mathrm{CH}) ; 129.0(\mathrm{CH}) ; 138.3 \\
(\mathrm{C}) ; 162.0(\mathrm{C}) ; 198.0(\mathrm{C}=\mathrm{O}) .\end{array}$ \\
\hline $6 f$ & 3 & 132 & 89 & $\begin{array}{l}\mathrm{C}_{15} \mathrm{H}_{19} \mathrm{NO} \\
229.32\end{array}$ & $\begin{array}{l}78.60 \\
78.51\end{array}$ & $\begin{array}{l}8.40 \\
8.35\end{array}$ & $\begin{array}{l}6.10 \\
6.11\end{array}$ & $\begin{array}{l}1.06(\mathrm{~s}, 6 \mathrm{H}) ; 2.15(\mathrm{~s}, 2 \mathrm{H}) ; \\
2.22(\mathrm{~s}, 2 \mathrm{H}) ; 4.21(\mathrm{~d}, J 6.0 \\
2 \mathrm{H}) ; 5.14(\mathrm{~s}, 1 \mathrm{H}) ; 5.20(\mathrm{br} \mathrm{s}, \\
1 \mathrm{H}, \mathrm{N}-\mathrm{H}) ; 7.27-7.31(\mathrm{br}, \\
\left.5 \mathrm{H}_{\text {arom }}\right)\end{array}$ & $\begin{array}{l}28.1\left(\mathrm{CH}_{3}\right) ; 32.7\left(\mathrm{CH}_{2}\right) ; 43.1(\mathrm{C}) ; \\
48.9\left(\mathrm{CH}_{2}\right) ; 50.2\left(\mathrm{CH}_{2}\right) ; 95.7(\mathrm{CH}) ; \\
127.3(\mathrm{CH}) ; 127.6(\mathrm{CH}) ; 128.6 \\
(\mathrm{CH}) ; 136.9(\mathrm{C}) ; 163.1(\mathrm{C}) ; \\
196.8(\mathrm{C}=\mathrm{O}) .\end{array}$ \\
\hline $6 \mathrm{~g}$ & 3 & - & 92 & $\begin{array}{l}\mathrm{C}_{11} \mathrm{H}_{17} \mathrm{NO} \\
179.26\end{array}$ & $\begin{array}{l}73.70 \\
73.38\end{array}$ & $\begin{array}{l}9.56 \\
9.43\end{array}$ & $\begin{array}{l}7.80 \\
7.79\end{array}$ & $\begin{array}{l}1.06(\mathrm{~s}, 6 \mathrm{H}) ; 2.17(\mathrm{~s}, 2 \mathrm{H}) ; \\
2.23(\mathrm{~s}, 2 \mathrm{H}) ; 3.73(\mathrm{br}, 2 \mathrm{H}) \\
5.10(\mathrm{~s}, 1 \mathrm{H}) ; 5.22(\mathrm{~m}, 2 \mathrm{H}) ; \\
5.42(\mathrm{br}, 1 \mathrm{H}, \mathrm{N}-\mathrm{H}) ; 5.84 \\
(\mathrm{~m}, 1 \mathrm{H}) .\end{array}$ & $\begin{array}{l}28.2\left(\mathrm{CH}_{3}\right) ; 28.4\left(\mathrm{CH}_{2}\right) ; 43.3(\mathrm{C}) ; \\
45.3\left(\mathrm{CH}_{2}\right) ; 50.0\left(\mathrm{CH}_{2}\right) ; 95.5(\mathrm{CH}) ; \\
117.4\left(\mathrm{CH}_{2}\right) ; 132.4(\mathrm{CH}) ; 163.5 \\
(\mathrm{C}) ; 197.0(\mathrm{C}=\mathrm{O}) .\end{array}$ \\
\hline
\end{tabular}


Preparation of the salts: ammonium acetate derivatives

Amine (2 equiv.) was added dropwise to acetic acid (1 equiv.) under ice cooling. The solid formed was filtered off and washed with diethyl ether, dried and immediately used. Yield 70-90\%.

4-Amino substituted-3-penten-2-ones (1a-f) and ethyl 3amino substituted-2-butenoates (2a-f), general procedure

Acetylacetone (for 1) or ethyl acetoacetate (for 2) ( $2 \mathrm{mmol}$ ), and the acetate of the corresponding amines (4 mmol) were dispersed on montmorillonite K-10 (0.60 g). The heterogeneous mixture was submitted to microwave irradiation for $3 \mathrm{~min}$ (except for 1-2a,b and $\mathbf{g}$ which was $5 \mathrm{~min}$ ) at $155 \mathrm{~W}$. The products were extracted by washing the $\mathrm{K}-10$ with $\mathrm{CH}_{2} \mathrm{Cl}_{2}$. The organic layer was washed with saturated $\mathrm{NaHCO}_{3}$, dried with $\mathrm{MgSO}_{4}$, filtered and the solvent was removed under vacuum to yield crude products.

Ethyl (3-amino substituted)-2-chloro-2-butenoates (4a-f), general procedure

Ethyl-2-chloro-acetoacetate $(5 \mathrm{mmol})$ and the acetate of the corresponding amines $(10 \mathrm{mmol})$ were dispersed on montmorillonite K-10 (1.50 g). The heterogeneous mixture was submitted to microwave irradiation for $3 \min$ (for $4 \mathbf{b} 2$ $\mathrm{min})$ at $155 \mathrm{~W}$. The products were extracted by washing the $\mathrm{K}-10$ with $\mathrm{CH}_{2} \mathrm{Cl}_{2}$. The organic layer was washed with saturated $\mathrm{NaHCO}_{3}$, dried with $\mathrm{MgSO}_{4}$, filtered and the solvent was removed under vacuum to yield a pure product, an oil at room temperature (Table 1).

\section{3-Amino substituted-5,5-dimethylcyclohex-2-enones (6a-g), general procedure}

5,5-Dimethylcyclohex-2-en-1-one $(2 \mathrm{mmol})$ and the corresponding amines (4 mmol) (for $\mathbf{6 a - c}, \mathbf{6} \mathbf{f}$ and $\mathbf{6 g}$ their acetate derivatives were used) were dispersed on montmorillonite K-10 $(0.60 \mathrm{~g})$. The heterogeneous mixture was submitted to microwave irradiation for the appropriate time (given in Table 2) at $155 \mathrm{~W}$. The products were extracted by washing the $\mathrm{K}-10$ with $\mathrm{CH}_{2} \mathrm{Cl}_{2}$. The organic layer was washed with saturated $\mathrm{NaHCO}_{3}$, dried with $\mathrm{MgSO}_{4}$, filtered and the solvent was removed under vacuum to yield crude product. Compounds 6 were purified by recrystallization from diisopropyl ether- $\mathrm{CH}_{2} \mathrm{Cl}_{2}$ (Table 2).

\section{Acknowledgments}

We are grateful to the Fundação de Amparo à Pesquisa do Estado do Rio Grande do Sul (FAPERGS) for financial support (01/0506.6). We thank to Dr. Robert Burrow for helpful comments on the manuscript.

\section{References}

1. Lidstrom, P.; Tierney, J.; Wathey, B.; Westman, J.; Tetrahedron 2001, 57, 9225.

2. Laszlo, P. ed.; Preparative Chemistry Using Supported Reagents Academic Press: New York, 1987.

3. Michael, J. P.; de Koning, C. B.; Gravestock, D.; Hosken, G. D.; Howard, A. S.; Jungman, C. M.; Krause, R. W. M.; Parsons, A. S.; Pelly, S. C.; Stambury, T. V.; Pure Appl. Chem. 1999, 71, 979.

4. Braibante, M. E. F.; Braibante, H. T. S.; Missio, L. J.; J. Heterocycl. Chem. 1996, 33, 1243; Braibante, M. E. F.; Braibante, H. T. S.; Valduga, C. J.; J. Heterocycl. Chem. 1997, 34, 1453; Braibante, M. E. F.; Braibante, H. T. S.; Valduga, C. J.; Santis, D. B.; J. Heterocycl. Chem. 1999, 36, 505; Braibante, M. E. F.; Braibante, H. T. S.; Costa, C. C.; Martins, D. B.; Tetrahedron Lett. 2002, 43, 8079; Greenhill, J. V.; Chaaban. I.; Stell, P. J.; J. Heterocycl. Chem. 1992, 29, 1375.

5. Cunha, S.; Rodovalho, W.; Azevedo, N. R.; Mendonça, M. O.; Lariucci, C.; Vencato, I.; J. Braz. Chem. Soc. 2002, 13, 629.

6. Kubicki, M.; Bassyouni, H. A. R.; Codding, P.W.; J. Mol. Struct. 2000, 525, 141.

7. Scott, K. R.; Edafiogho, I. O.; Richardson, E. L.; Farrar, V. A.; Moore, J. A.; Tietz, E. I.; Hinko, C. N. Chang, H.; El-Assadi, A.; Nicholsonn, J. M.; J. Med. Chem. 1993, 36, 1947.

8. Greenhill, J. V.; Chem. Soc. Rev. 1977, 6, 277.

9. Alberola, A.; Andres, C.; Ortega, A. G.; Pedrosa, R.; Vicente, M.; An. Quim. 1987, 83, 55.

10. Cone, E. J.; Garner, R. H.; Hayes, A. W.; J. Org. Chem. 1972, $37,4436$.

11. Braibante, M. E. F.; Braibante, H. T. S.; Missio, L .J.; Andricopulo, A.; Synthesis 1994, 898.

12. Braibante, M. E. F.; Braibante, H. T. S.; Valduga, C.; Squizani, A.; Synthesis 1998, 1019.

13. Texier-Boullet, F.; Rechsteiner, B.; Hamelin, J.; Tetrahedron Lett. 1993, 34, 5071.

14. Kuckländer, V. In The Chemistry of Enamines; Part 1; Rappoport, Z. ed., John Wiley \& Sons: New York, 1994; pp. 525-639. 